\title{
Sudden death in epilepsy and ectopic neurohypophysis in Joubert syndrome 23 diagnosed using SNVs/indels and structural variants pipelines on WGS data: a case report
}

Dulika Sumathipala ${ }^{1 \dagger}$, Petter Strømme ${ }^{2,3 \dagger}$, Christian Gilissen ${ }^{4}$, Ingunn Holm Einarsen ${ }^{1}$, Hilde J. Bjørndalen², Andrés Server ${ }^{5}$, Jordi Corominas ${ }^{4}$, Bjørnar Hassel ${ }^{3,6}$, Madeleine Fannemel ${ }^{1}$, Doriana Misceo ${ }^{1 *+}$ and Eirik Frengen ${ }^{1+}$

\begin{abstract}
Background: Joubert syndrome (JBTS) is a genetically heterogeneous group of neurodevelopmental syndromes caused by primary cilia dysfunction. Usually the neurological presentation starts with abnormal neonatal breathing followed by muscular hypotonia, psychomotor delay, and cerebellar ataxia. Cerebral MRI shows mid- and hindbrain anomalies including the molar tooth sign. We report a male patient with atypical presentation of Joubert syndrome type 23, thus expanding the phenotype.

Case presentation: Clinical features were consistent with JBTS already from infancy, yet the syndrome was not suspected before cerebral MRI later in childhood showed the characteristic molar tooth sign and ectopic neurohypophysis. From age 11 years seizures developed and after few years became increasingly difficult to treat, also related to inadequate compliance to therapy. He died at 23 years of sudden unexpected death in epilepsy (SUDEP). The genetic diagnosis remained elusive for many years, despite extensive genetic testing. We reached the genetic diagnosis by performing whole genome sequencing of the family trio and analyzing the data with the combination of one analysis pipeline for single nucleotide variants (SNVs)/indels and one for structural variants (SVs). This lead to the identification of the most common variant detected in patients with JBTS23 (OMIM\# 616490), rs534542684, in compound heterozygosity with a $8.3 \mathrm{~kb}$ deletion in KIAA0586, not previously reported.

Conclusions: We describe for the first time ectopic neurohypophysis and SUDEP in JBTS23, expanding the phenotype of this condition and raising the attention on the possible severity of the epilepsy in this disease. We also highlight the diagnostic power of WGS, which efficiently detects SNVs/indels and in addition allows the identification of SVs.
\end{abstract}

Keywords: Case report, Ectopic neurohypophysis, Epilepsy, Joubert syndrome, KIAA0586, WGS

\footnotetext{
* Correspondence: doriana.misceo@medisin.uio.no

${ }^{\dagger}$ Dulika Sumathipala and Petter Strømme contribute equally.

Doriana Misceo and Eirik Frengen contributed equally.

'Department of Medical Genetics, Oslo University Hospital and University of

Oslo, Oslo, Norway

Full list of author information is available at the end of the article
}

(c) The Author(s). 2020 Open Access This article is licensed under a Creative Commons Attribution 4.0 International License, which permits use, sharing, adaptation, distribution and reproduction in any medium or format, as long as you give appropriate credit to the original author(s) and the source, provide a link to the Creative Commons licence, and indicate if changes were made. The images or other third party material in this article are included in the article's Creative Commons licence, unless indicated otherwise in a credit line to the material. If material is not included in the article's Creative Commons licence and your intended use is not permitted by statutory regulation or exceeds the permitted use, you will need to obtain permission directly from the copyright holder. To view a copy of this licence, visit http://creativecommons.org/licenses/by/4.0/. The Creative Commons Public Domain Dedication waiver (http://creativecommons.org/publicdomain/zero/1.0/) applies to the data made available in this article, unless otherwise stated in a credit line to the data. 


\section{Background}

Joubert syndrome (JBTS, OMIM\# 213300) [1] is a rare disease with autosomal recessive inheritance, caused by the dysfunction of primary cilia and presenting as a neurodevelopmental syndrome [2]. Typical manifestations of the disease include neonatal breathing anomalies, hypotonia, cognitive impairment, cerebellar ataxia, and a complex mid- and hind-brain malformation visible as a molar tooth sign on axial cerebral MRI scans, which is a diagnostic signature finding in JBTS. Non-neurological manifestations, including cystic kidneys, liver disease, retinal dystrophy, chorioretinal colobomas, and polydactyly, may also be present. The disease is genetically heterogeneous with more than 35 genes currently known to cause it when mutated [3]. Among those genes, KIAA0586 (OMIM\# 610178) has been documented to cause JBTS23 (OMIM\# 616490). Biallelic variants in KIAA0586 are responsible of $2.5-7 \%$ of all patients with JBTS [4, 5]. In addition to JBTS23, dysfunction of KIAA0586 may cause a more severe ciliopathy known as Short-rib thoracic dysplasia 14 with polydactyly (SRTD14; OMIM\# 616546), a complex syndrome with skeletal and neurological manifestations [6].

The KIAA0586 protein plays a central role in cilia formation. Primary cilia function as cellular antennae and are required for several signaling pathways essential for tissue growth and differentiation. KIAA0586 is a centrosomal protein, located at the distal ends of both the mother and daughter centrioless [7]. During cilia formation, KIAA0586 is crucial for the maturation of the mother centriole, through centriolar satellite dispersal and assembly of the basal body distal appendages, and basal body docking to the plasma membrane [8]. KIAA0586 functions upstream of the small GTPase Rab8 required for docking of the basal body to the plasma membrane $[8,9]$.

We present a Norwegian male patient with JBTS23. The genetic diagnosis was reached by performing whole genome sequencing (WGS) of the family trio, and analyzing the data with two distinct analysis pipelines one for single nucleotide variants (SNVs)/indels and one for structural variants (SVs). This allowed us to identify two pathogenic variants in KIAA0586 and give the patient the genetic diagnosis of JBTS23. The patient developed epilepsy and died in early adulthood. Cerebral MRI revelead the presence of ectopic neurohypophisis, which has not been previously reported in JBTS23. We therefore expand the clinical phenotype and highlight the possible severity of epilepsy in JBTS23.

\section{Case presentation}

We report a male patient who was the younger of two siblings born to non-consanguineous parents from Norway. The elder sibling and his parents were healthy.
Initially, the patient presented with infantile episodes of apnea and tachypnea with hypotonia and global developmental delay. He manifested abnormal eye movements with oculomotor apraxia and Duane anomaly (Fig. 1), and cerebellar ataxia. Cerebral MRI showed the "molar tooth sign" characterized by elongated, thickened superior cerebellar peduncles, vermian hypoplasia and abnormal deep interpeduncular fossa (Fig.2a-d), neuroanatomical hallmarks of JBTS as well as ectopic neurohypophysis (Fig.2e-f). He had learning difficulties and when tested with a Wechsler Intelligence Scale for Children (WISC) he obtained a score of IQ 72. At 11 years he was diagnosed with epileptic seizures, at first only occurring at night, and had therefore escaped recognition for almost a year. He was admitted for the first time at 11 years and 9 months with a nocturnal generalized tonic clonic seizure (GTCS) and was started on Oxcarbazepine, which kept him almost seizure free for several years. This drug was tapered at $18 \mathrm{y}$, but soon after his epilepsy recurred, now manifesting both as GTCS and absences. Oxcarbazepine was reinstituted, but absences, sometimes with myoclonus, persisted, and treatment was changed to Valproate. However, GTCS increased in frequency, often followed by prolonged headaches that did not clear before the next day. His treatment was then changed to Lamotrigine, which he however was reluctant to take. He was living alone and managed to attend shelter work. At 22 years he was recognized to have two seizure types, GTCS and sensory epilepsy, the latter manifesting as abrupt abdominal discomfort, considered to be of temporal lobe origin. Nocturnal GTCS occurred 2-3 times per week and were often initiated by yawning and drooling and ending with vomiting, followed by drowsiness for the rest of the day. At 23 years one morning he was found dead in bed and according to his mother he laid in a flexed position that was usual for him after having a seizure. Postmortem examination concluded with no trace of Lamotrigine in his blood. The fatal outcome was classified as sudden unexpected death in epilepsy (SUDEP) [10], and was suspected to have been precipitated by the lack of compliance to anticonvulsive treatment.

\section{Genetic investigations}

Microarray and whole exome sequencing (WES) data analysis of the family trio did not identify any putative pathogenic variants explaining the disease (data not shown); therefore, we performed WGS of the family trio (methods are described in the Additional file 1). We reached the genetic diagnosis of the patient combining two WGS data analysis pipelines. First, we analyzed SNVs/indels, which were called on the patient-parent trio data using Genome Analysis Toolkit (GATK) v.3.4. Haplotype Caller in GVCF model. The first analysis with 

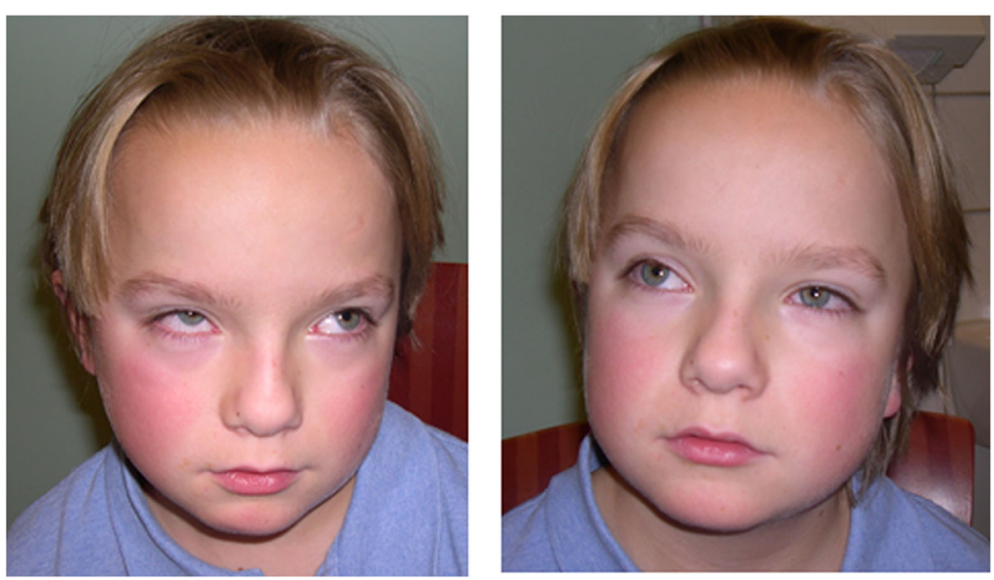

Fig. 1 Photograph of the patient at the age of 11 years showing Duane (retraction) anomaly: when gazing to the left, the globe of the adducting right eye is retracting and when gazing to the right, the globe of the adducting left eye is retracting. Lateral eye movement to either side is limited because the corresponding abducens nerve nucleus inadequately innervates the lateral rectus muscle, resulting in globe retraction and narrowing of the palpebral fissure

this pipeline identified a maternally inherited frameshift variant in KIAA0586 (NM_001244189), chr14 (GRCh37): g.58899157delG (c.428delG) (Fig. 3a), rs534542684, predicted to form a premature stop codon (p.Arg143Lysfs*4), which was verified by Sanger sequencing. This variant, was previously reported as pathogenic [5, 11-13]. Pathogenic variants in KIAA0586 cause two distinct autosomal recessive diseases [4-7, 11-13]. One is the Short-rib thoracic dysplasia 14 with polydactyly (SRTD14; OMIM\# 616546), a complex syndrome with skeletal and neurological manifestations [6]. The other is JBTS23 (OMIM\# 616490). As the variant could not explain the recessive phenotype, we subsequently used a data analysis pipeline for structural variants (details in the Supplementary information), which detected a paternally inherited $8.3 \mathrm{~kb}$ deletion in chr14 (GRCh37):g.58910278-58918611 according to variant calling of the high throughput sequencing data. The deletion lead to removal of exons 8, 9 and 10 in KIAA0586 (NM_ 001244189), predicted to cause direct splicing of exon 7 to 11 in the transcript, leading to a frameshift and formation
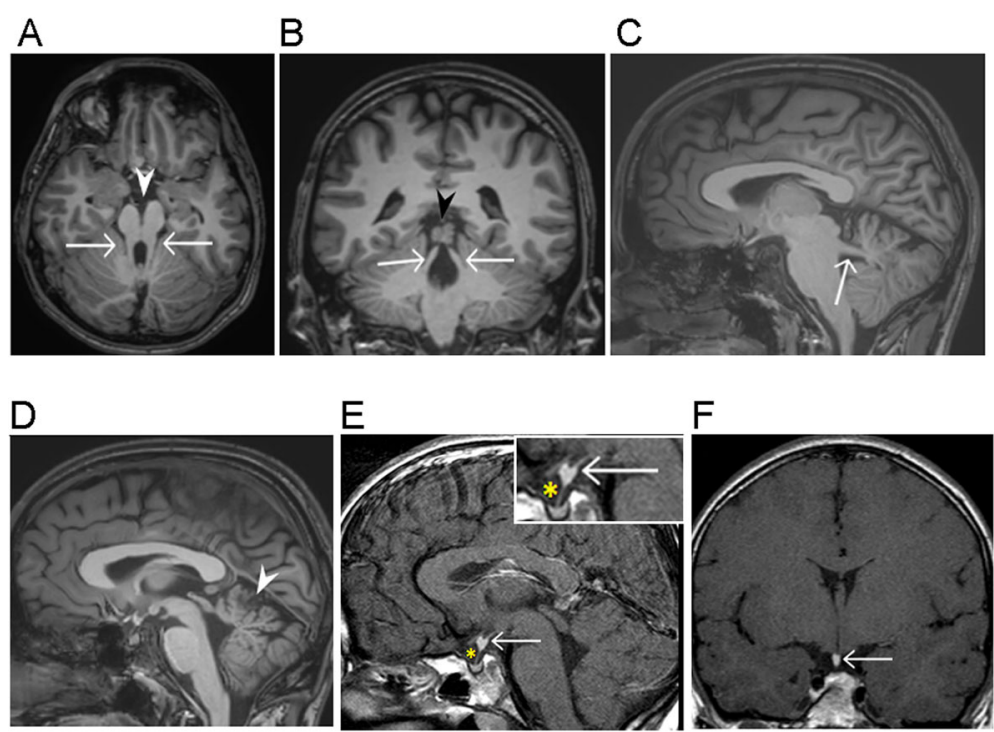

Fig. 2 a-d Molar tooth sign in JBTS. Axial (a), coronal (b) and sagittal (c) T1-weighted images show large, thickened, elongated superior cerebellar peduncles (arrows). Note a deep interpeduncular fossa (white arrowhead) and the vermian cleft (black arrowhead). Midsagittal (d) T1-weighted image shows vermian hypoplasia (white arrowhead). e-f Ectopic posterior pituitary lobe. Postcontrast sagittal and coronal T1-weighted images show a posterior pituitary lobe (arrow) located at the level of the upper infundibulum. A very thin infundibulum is seen (asterisk) (magnified in the right upper corner of $\mathrm{E})$. 

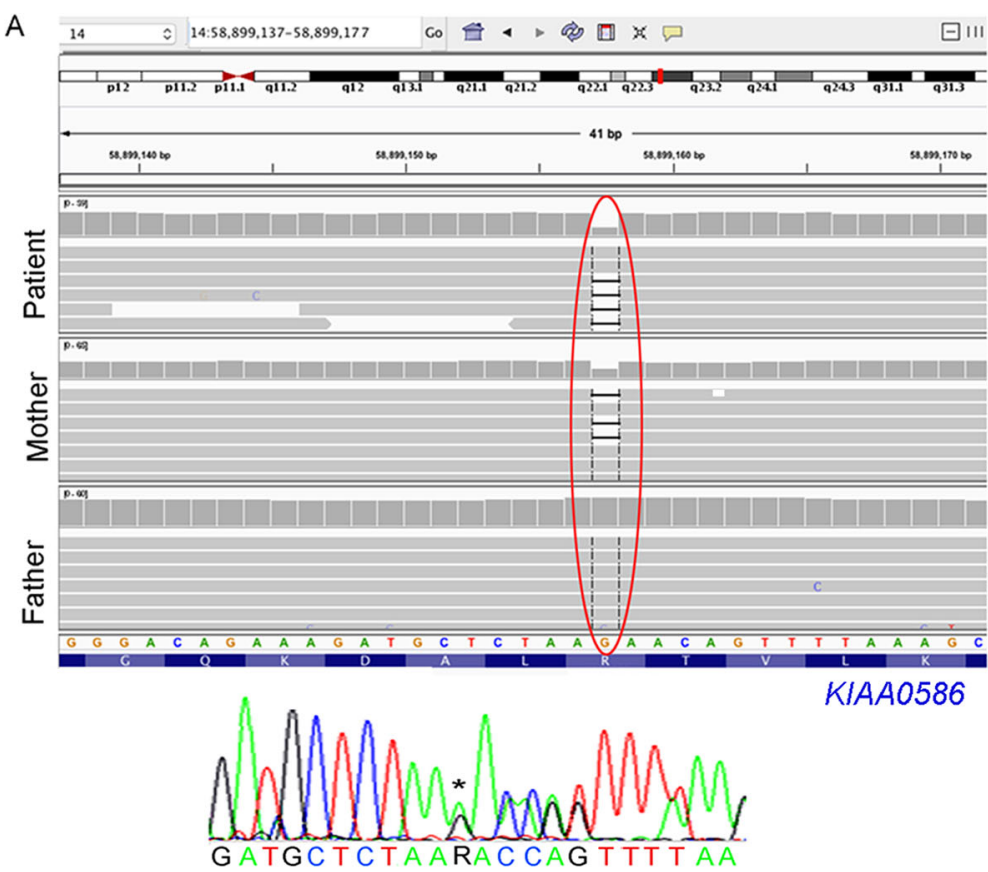

KIAA0586
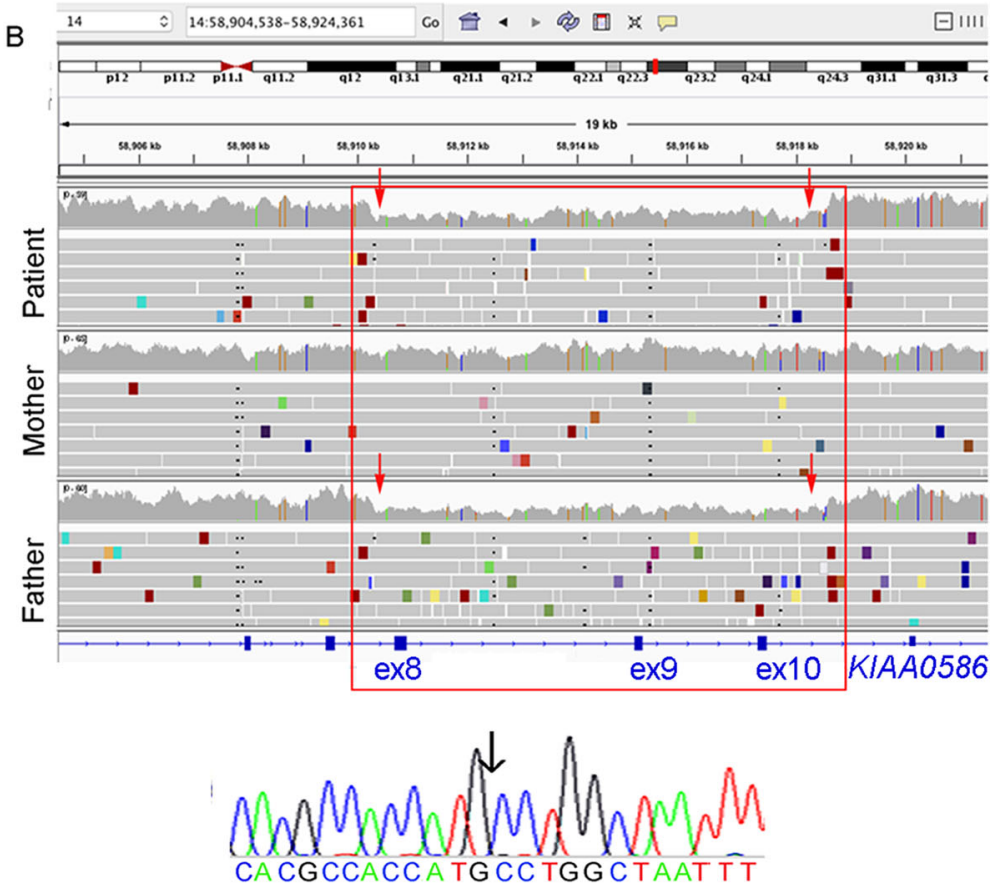

Fig. 3 a Upper. WGS data in Integrative Genome Viewer (IGV) (http://software.broadinstitute.org/software/igv) showing the heterozygous 1 bp deletion in KIAA0586 in the patient and his mother, but not in the father (red oval). Lower. Sanger sequencing on DNA from blood of the patient verifying 1 bp deletion in KIAA0586. $\mathrm{R}=\mathrm{A}$ or $\mathrm{G}$ (as a consequence of the heterozygous deletion of $\mathrm{G}$ followed by A). $\mathbf{b}$ Upper. Screenshot from the IGV showing the $8.3 \mathrm{~kb}$ deletion in KIAA0586 detected in heterozygosity in the patient and his father, but not in the mother (red square) removing exons 8, 9 and 10 in KIAA0586 (blue boxes in the bottom). Note the decreased coverage in the deleted region in the patient and his father (red vertical arrows) and the reads with ends spanning the deletions (in red). Lower. Sanger sequencing of DNA from blood of the patient verified the $8.3 \mathrm{~kb}$ deletion in KIAA0586 and re-defined its breakpoints slightly to chr14 (GRCh37):g.58910301-58,918,610.

of a premature stop codon (p.Val249Glufs*3). Deletions of this genomic region has not previously been described (Database of Genomic Variants, http://dgv.tcag.ca and
Decipher, https://decpher.sanger.ac.uk). Sanger sequencing analysis verified the segregation of this deletion with the disease in the family and re-defined the breakpoints of the 
SV slightly to chr14 (GRCh37):g.58910301-58918610 (Fig. $3 \mathrm{~b})$. The discrepancy in the break-end calculation can be attributed to the Manta base-pair resolution capability [14]. The clinical presentation of the patient was compatible with JBTS23. Thus, we identified pathogenic compound heterozygous variants in KIAA0586 by applying two distinct analysis pipelines on the WGS data, and confirmed the patient with JBTS23. We did not identify additional deleterious variants in other known JBTS causing genes in WES or WGS data.

\section{Discussion and conclusions}

We report a patient with biallelic variants in KIAA0586, causing JBTS23. The maternal KIAA0586 variant, c.428delG, known as rs534542684, is reported with minor allele frequency (MAF) 0.003 in the Exome Aggregate Consortium (ExAC) and in the Genome Aggregation database (gnomAD). Surprisingly, this variant has been identified in homozygosity in two healthy individuals in the European population in gnomAD and in one healthy homozygous female. In this female the KIAA0586 c.428delG transcript was shown to elude the nonsense-mediated decay (NMD) mechanism [12]. It was suggested that the allele carrying rs534542684 could function as a hypomorphic allele, possibly by the use of an alternative start codon downstream of the variant [12]. It is interesting to note that c.428delG is the most frequently identified variant in JBTS23, usually present in compound heterozygosity, less often in homozygosity $[5,13]$. In our patient the second allele harbored a deletion removing exons $8-10$.

Patients with JBTS23 present with neonatal breathing pattern anomalies, global developmental delay, intellectual disability, and brain malformations, including the molar tooth sign. In addition to the typical JBTS23 presentation, our patient manifested with Duane anomaly, previously reported only in one case [13]. Interestingly, the patient suffered from epilepsy and had an ectopic neurohypophysis. The last feature has never been reported in patients with JBTS23.

Epilepsy is reported only in patients with mutations in five of the known JBTS causing genes: CC2D2A causing JBTS9 (OMIM\# 612285), KIF7 causing JBTS12 (OMIM\# 200990), KIAA0586 causing JBTS23 (OMIM\# 616490), ARMC9 causing JBTS30 (OMIM\# 617622), and B9D2 causing (OMIM\# 614175). None of the animal models targeting KIAA0586 orthologues so far reported manifested epilepsy [15]. Among the 42 JBTS23 patients so far reported $[4,5,7,11-13]$, only two suffered from epilepsy [7, 11], and SUDEP has not been reported. However, the patient presented several clinical risk factors for the occurrence of SUDEP, such as being a male with a history of seizures from a young age, often nocturnal GTCS, borderline intellectual disability, and living alone not complying to the treatment [10]. In particular, the combination of frequent nocturnal GTCS and sleeping alone was showed to dramatically increase the risk of SUDEP [16]. In general mortality in JBTS has not been related to epilepsy, but rather to renal or respiratory failure [17], however the recent management recommendations for patients with JBTS specifically mention seizures [3].

Our patient is the first to be reported with deleterious variants in KIAA0586 and ectopic neurohypophysis. The ectopic neurohypophysis is a midline brain malformation consisting of an aberrant pituitary development with an ectopically located posterior pituitary gland. This brain malformation can be associated with endocrinological defects ranging from isolated growth hormone deficiency to multiple anterior pituitary hormone deficiencies, but posterior pituitary function remains unchanged. However, our patient had a normal endocrinological profile. It was proposed that the occurrence of the ectopic neurohypophysis in ciliopathies might be caused by the role of the cilia in influencing pituitary development through SHH and Wnt signaling pathways [18].

In conclusion, we identified compound heterozygous variants in KIAA0586 in a patient with JBTS23, presenting with ectopic neurohypophysis and juvenile onset of epilepsy, which was difficult to treat and resulted in SUDEP. We expand the phenotype of JBTS23 since these features have not previously been reported in this disease. Although we report a single patient, we suggest that the onset of epilepsy in patients with JBTS23 should be promptly evaluated and treated, and deserve close monitoring, to reduce the risk of an adverse outcome. Our results highlight the value of WGS when combining different pipelines of analysis to detect $\mathrm{SNV}$ /indels and larger structural variants below the resolution of diagnostic microarrays.

\section{Supplementary information}

Supplementary information accompanies this paper at https://doi.org/10. 1186/s12881-020-01024-y.

Additional file 1. Details of the methods for the genetic investigations.

\footnotetext{
Abbreviations

IGV: Integrative genome viewer; JBTS: Joubert syndrome; OMIM: Online Mendelian inheritance in men; SNVs: Single nucleotide variants; SUDEP: Sudden unexpected death in epilepsy; SVs: Structural variants; WES: Whole exome sequencing; WGS: Whole genome sequencing

Acknowledgments

We thank the family for participating to the study. The genome sequencing service was provided by the Norwegian High-Throughput Sequencing Centre, a national technology platform supported by the 'Functional Genomics' and by the 'Infrastructure' programs of the Research Council of Norway and the Southeastern Regional Health Authorities. We thank UNINETT Sigma2, the National Infrastructure for High Performance Computing and Data Storage in Norway, for support to store and analyze HTS data.
} 


\section{Authors' contributions}

DS: Conceptualization; Data curation; Formal analysis; Methodology; Visualization; Writing-original draft; Writing-review \& editing. PS: Conceptualization; Data curation; Clinical Investigation; Supervision; Writingoriginal draft; Writing-review \& editing. CG and JC: Data curation; Formal analysis; Methodology. IHE: Data curation; Validation; Visualization. BH, HJB, AS, MF, Clinical Investigations. DM: Conceptualization; Methodology; Supervision; Visualization; Writing-original draft; Writing-review \& editing. EF: Conceptualization; Methodology; Supervision; Writing-review \& editing; Project administration. All authors read and approved the manuscript.

\section{Funding}

The Norwegian National Advisory Unit on Rare Diseases (Nasjonal kompetansetjeneste for sjeldne diagnoser, NKSD) (https://oslouniversitetssykehus.no/fag-og-forskning/nasjonale-og-regionale-tjenester/ nasjonal-kompetansetjeneste-for-sjeldne-diagnoser) provided funding to PS for genome sequencing. DS was supported financially by the Quota scheme of Norwegian State Educational Loan Fund, and mobility grants from EMBO. DM and DS were supported by UNIFOR, University of Oslo. The funders had no role in study design, data collection and analysis decision to publish or preparation of the manuscript.

\section{Availability of data and materials}

Data available on request, but may be subjected to restrictions due to privacy/ethical restrictions.

\section{Ethics approval and consent to participate}

Written informed consent to participate was obtained from the parents of the patient. The study was approved by the Regional Committee for Medical Research Ethics - South-East Norway, REK 2010/1152a.

\section{Consent for publication}

Written consent for the publication of this case report was obtained by the parents of the patient.

\section{Competing interests}

The authors declare that they have no competing interests.

\section{Author details}

${ }^{1}$ Department of Medical Genetics, Oslo University Hospital and University of Oslo, Oslo, Norway. ${ }^{2}$ Division of Pediatric and Adolescent Medicine, Oslo University Hospital, Oslo, Norway. ${ }^{3}$ Faculty of Medicine, University of Oslo, Oslo, Norway. ${ }^{4}$ Department of Human Genetics, Radboud UMC, Nijmegen, The Netherlands. ${ }^{5}$ Section of Neuroradiology, Department of Radiology and Nuclear Medicine, Oslo University Hospital, Rikshospitalet, Oslo, Norway. ${ }^{6}$ Department of Neurohabilitation and Complex Neurology, Oslo University Hospital, Ullevål, Oslo, Norway.

Received: 27 December 2019 Accepted: 12 April 2020

Published online: 07 May 2020

\section{References}

1. Parisi MA. Clinical and molecular features of Joubert syndrome and related disorders. Am J Med Genet C Semin Med Genet. 2009;151C(4):326-40.

2. Mitchison HM, Valente EM. Motile and non-motile cilia in human pathology: from function to phenotypes. J Pathol. 2017;241(2):294-309.

3. Bachmann-Gagescu R, Dempsey JC, Bulgheroni S, Chen ML, D'Arrigo S, Glass IA, et al. Healthcare recommendations for Joubert syndrome. Am J Med Genet A. 2020;182(1):229-49.

4. Vilboux T, Doherty DA, Glass IA, Parisi MA, Phelps IG, Cullinane AR, et al. Molecular genetic findings and clinical correlations in 100 patients with Joubert syndrome and related disorders prospectively evaluated at a single center. Genet Med. 2017;19(8):875-82.

5. Bachmann-Gagescu R, Phelps IG, Dempsey JC, Sharma VA, Ishak GE, Boyle EA, et al. KIAA0586 is mutated in Joubert syndrome. Hum Mutat. 2015;36(9): 831-5.

6. Alby C, Piquand K, Huber C, Megarbane A, Ichkou A, Legendre M, et al. Mutations in KIAA0586 cause lethal Ciliopathies ranging from a Hydrolethalus phenotype to short-rib Polydactyly syndrome. Am J Hum Genet. 2015;97(2):311-8.
7. Stephen LA, Tawamie H, Davis GM, Tebbe L, Nurnberg P, Nurnberg G, et al. TALPID3 controls centrosome and cell polarity and the human ortholog KIAA0586 is mutated in Joubert syndrome (JBTS23). Elife. 2015;4:e08077.

8. Kobayashi T, Kim S, Lin YC, Inoue T, Dynlacht BD. The CP110-interacting proteins Talpid3 and Cep290 play overlapping and distinct roles in cilia assembly. J Cell Biol. 2014;204(2):215-29.

9. Ojeda Naharros I, Cristian FB, Zang J, Gesemann M, Ingham PW, Neuhauss SCF, et al. The ciliopathy protein TALPID3/KIAA0586 acts upstream of Rab8 activation in zebrafish photoreceptor outer segment formation and maintenance. Sci Rep. 2018;8(1):2211.

10. Jones $L A$, Thomas $R H$. Sudden death in epilepsy: insights from the last 25 years. Seizure. 2017:44:232-6.

11. Malicdan MC, Vilboux T, Stephen J, Maglic D, Mian L, Konzman D, et al. Mutations in human homologue of chicken talpid3 gene (KIAA0586) cause a hybrid ciliopathy with overlapping features of Jeune and Joubert syndromes. J Med Genet. 2015;52(12):830-9.

12. Pauli S, Altmuller J, Schroder S, Ohlenbusch A, Dreha-Kulaczewski S, Bergmann C, et al. Homozygosity for the c.428delG variant in KIAA0586 in a healthy individual: implications for molecular testing in patients with Joubert syndrome. J Med Genet. 2019;56(4):261-4.

13. Roosing S, Hofree M, Kim S, Scott E, Copeland B, Romani M, et al. Functional genome-wide siRNA screen identifies KIAA0586 as mutated in Joubert syndrome. Elife. 2015;4:e06602.

14. Chen X, Schulz-Trieglaff $O$, Shaw R, Barnes B, Schlesinger F, Kallberg M, et al. Manta: rapid detection of structural variants and indels for germline and cancer sequencing applications. Bioinformatics. 2016;32(8):1220-2.

15. Fraser AM, Davey MG. TALPID3 in Joubert syndrome and related ciliopathy disorders. Curr Opin Genet Dev. 2019:56:41-8.

16. Sveinsson O, Andersson T, Mattsson P, Carlsson S, Tomson T. Clinical risk factors in SUDEP: a nationwide population-based case-control study. Neurology. 2020;94(4):e419-e29.

17. Dempsey JC, Phelps IG, Bachmann-Gagescu R, Glass IA, Tully HM, Doherty D. Mortality in Joubert syndrome. Am J Med Genet A. 2017;173(5):1237-42.

18. Khonsari RH, Seppala M, Pradel A, Dutel H, Clement G, Lebedev O, et al. The buccohypophyseal canal is an ancestral vertebrate trait maintained by modulation in sonic hedgehog signaling. BMC Biol. 2013;11:27.

\section{Publisher's Note}

Springer Nature remains neutral with regard to jurisdictional claims in published maps and institutional affiliations.
Ready to submit your research? Choose BMC and benefit from:

- fast, convenient online submission

- thorough peer review by experienced researchers in your field

- rapid publication on acceptance

- support for research data, including large and complex data types

- gold Open Access which fosters wider collaboration and increased citations

- maximum visibility for your research: over $100 \mathrm{M}$ website views per year

At $\mathrm{BMC}$, research is always in progress.

Learn more biomedcentral.com/submissions 